\title{
Novel and effective synthesis protocol of AgNPs functionalized using L-cysteine as a potential drug carrier
}

\author{
Marek Wojnicki $^{1} \cdot$ Magdalena Luty-Błocho $^{1} \cdot$ Magdalena Kotańska $^{2} \cdot$ Magdalena Wytrwal $^{3} \cdot$ Tomasz Tokarski $^{3}$. \\ Anna Krupa $^{4} \cdot$ Marcin Kolaczkowski $^{5} \cdot$ Adam Bucki $^{5} \cdot$ Marcin Kobielusz $^{6}$
}

Received: 8 August 2017 / Accepted: 8 November 2017 / Published online: 16 November 2017

(C) The Author(s) 2017. This article is an open access publication

\begin{abstract}
In this study, the protocol of a single-step L-cysteine functionalized silver nanoparticle synthesis was described. Particle size distribution was determined. The crystallinity and chemical properties were investigated using XRD, HR-TEM, and XPS methods. Acute toxicity and irritant properties of obtained nanoparticles were studied using mice and rats as an animal model. The results showed that thanks to the applied protocol, it was possible to synthesize silver nanoparticles with narrow particle size distribution. Moreover, the concentration of final product was extremely high in comparison to other known methods. These nanoparticles showed neither irritant properties nor acute toxicity.
\end{abstract}

Marek Wojnicki

marekw@agh.edu.pl

1 Faculty of Non-Ferrous Metals, AGH University of Science and Technology, Al. A. Mickiewicza 30, 30-059 Krakow, Poland

2 Department of Pharmacological Screening, Chair of Pharmacy, Jagiellonian University, Medical College, 9 Medyczna Street, 30-688 Krakow, Poland

3 Academic Centre for Materials and Nanotechnology, AGH University of Science and Technology, al. A. Mickiewicza 30, 30-059 Krakow, Poland

4 Department of Pharmaceutical Technology and Biopharmaceutics, Jagiellonian University Medical College, 9 Medyczna Street, 30-688 Krakow, Poland

5 Department of Medicinal Chemistry, Jagiellonian University, Medical College, 9 Medyczna Street, 30-688 Krakow, Poland

6 Faculty of Chemistry, Jagiellonian University in Kraków, ul. Ingardena 3, 30-060 Krakow, Poland
Keywords Silver nanoparticles $\cdot$ Functionalization . L-cysteine $\cdot$ Toxicity $\cdot$ Drug carrier $\cdot$ Synthesis

\section{Introduction}

Throughout the last decades, the nanotechnology has been considered as a powerful field, which finds out the application in many areas of science. The progress of nanotechnology is especially important in clinical medicine, where development of new drugs, devices, and diagnostic tools improves our life. In medical application, particular noble metals, such as gold, platinum, palladium, and silver nanoparticles are considered, due to their unique chemical and physical properties. These particles have low toxicity, high compatibility to biological systems, and can be easily functionalized, which increase the range of their potential application. For these reasons, gold nanoparticles are used, e.g., in cancer therapy mainly as drug carriers (Cobley et al. 2011), delivery vehicles (Galdiero et al. 2011), or markers for imaging (Huang et al. 2006) etc. (Guo and Sadler 1999) Due to their antibacterial properties, silver nanoparticles have been used mostly in healthcare industry, food storage, textile manufacturing, cosmetics (Kokura et al. 2010), and environmental remediation. It is worth noting, however, that the application of nanoparticles is strictly dependent on their morphology (size, shape, and their size distribution), which can be easily manipulated by the use of a suitable manufacturing technique. For example, chemical methods allow to synthetize nanoparticles of different morphology (Luty-Błocho et al. 2014; Luty-Błocho and Wojnicki 2015). Among them, only a few synthesis routes are recommended for medical application as there are problems with the biocompatibility of reagents, or media, etc. Thus, in the present study, chemical reduction of metal precursor with reductant was chosen as a method for the synthesis 
of silver nanoparticles. The main advantages of this technique are low cost, simplicity, and the opportunity to synthetize the nanoparticles which size around $9 \mathrm{~nm}$. Dimethylamine borane was used as an effective reducing agent of silver ions (Wojnicki et al. 2015). In order to exclude a negative effect coming from products of nanoparticle synthesis, the solution after redox reaction was dialyzed (see the "Silver nanoparticle synthesis and characterization" section for more details). This method allows to remove all dangerous agents. In this paper, the special attention was focused on the functionalization of silver nanoparticles for cosmetic application.

\section{Materials and methods}

\section{Silver nanoparticle synthesis and characterization}

Silver nanoparticle synthesis was performed using silver(I) nitrate $(\mathrm{V})\left(\mathrm{AgNO}_{3}\right.$, Avantor Materials) as precursor. Borane dimethylamine complex of $97 \%$ (DMAB, Fluka) was used as the reducing agent. L-cysteine was used as stabilizer as well as functionalizing compound. AgNPs were synthesized as follows: $0.2 \mathrm{M}$ aqueous solution of $\mathrm{AgNO}_{3}$ was mixed in a 1:1 ratio with the aqueous solution containing $0.4 \mathrm{M}$ of L-cysteine and $1 \mathrm{M}$ of sodium hydroxide (Avantor Materials). Such a solution has lightly yellow color due to the formation of Lcysteine- $\mathrm{Ag}^{+}$complex. Then, freshly prepared aqueous solution containing $0.3 \mathrm{M}$ DMAB in $0.1 \mathrm{M} \mathrm{NaOH}$ was mixed in a $1: 1$ volumetric ratio with $\mathrm{L}-\mathrm{cysteine}-\mathrm{Ag}^{+}$containing solution. After mixing, the color of the solution was gradually changing from light yellow by red to dark brown.

It is worth to mention here that increase of DMAB initial concentration affects the decrease of particle size. However, there is no linear correlation between reductant concentration and particle size.

The dialysis method was applied to remove the residues after redox reaction. For this purpose, the Medical International Size 9 Int. Dia. 36/32" dialysis tubing was applied. The dialysis was performed for ca. 5 days. The continuous water exchange was set at $20 \mathrm{~mL} / \mathrm{min}$. Thanks to that, the $\mathrm{pH}$ level decreased to ca. 7. Also, the specific odor of dimethylamine was removed. Then, the solution was filtered using a Whatman PC MB membrane of $47 \mathrm{~mm}$ in diameter and the pore size of $50 \mathrm{~nm}$. This solution was used for further analysis and experiments.

Zeta potential as well as particle size distribution of the obtained composite material was determined using a Zetasizer Nano ZS (Malvern). A standard clear polycarbonate cell with gold electrodes was applied.

UV-Vis spectra were registered using a Shimadzu model U-2501 PC and quartz cuvette of $10 \mathrm{~mm}$ path length.

Fluorescence spectra were measured using a PerkinElmer LS55 spectrofluorometer in a $1 \mathrm{~cm}$ quartz cuvette.
HR-TEM analysis was performed using HR-TEM-FEI TECNAI TF 20 X-TWIN. One drop of freshly prepared colloidal suspension was placed on a copper grid covered with a 20-30-nm amorphous carbon film. Then, the sample was left to dry at room temperature (ca. $21^{\circ} \mathrm{C}$ ).

Particle size and size distribution were determined after analyzing of HR-TEM images using ImageJ application (ver. $1.48 \mathrm{~b}$ ).

$\mathrm{X}$-ray photoelectron spectroscopy measurements were performed using a PHI 5000 Versa Probe II (ULVAC-PHI, Chigasaki, Japan) system using a microfocused $(100 \mu \mathrm{m}$, $25 \mathrm{~W}) \mathrm{Al} \mathrm{K} \alpha \mathrm{X}$-ray beam with a photoelectron take-off angle of $45^{\circ}$. To compensate the charging effect, a dual-beam charge neutralizer was used. The operating pressure in the analytical chamber was less than $5 \times 10^{-7} \mathrm{~Pa}$. High-resolution spectra were collected with analyzer pass energy of $23.50 \mathrm{eV}$. XPS spectra were acquired from $400 \times 300 \mu \mathrm{m}$ (Galdiero et al. 2011) areas. All XPS peaks were referenced to the neutral (C-C) carbon C 1s peak at $284.8 \mathrm{eV}$. Spectrum background was subtracted using the Shirley method. Data analysis software from PHI MultiPak was used to calculate elemental compositions from the peak areas.

\section{Animal studies}

Adult female Albino Swiss mice weighing 28-33 g were used in the study. They were kept in environmentally controlled rooms, in standard cages lit by an artificial light for $12 \mathrm{~h}$. Animals had free access to food and water. The randomly established experimental groups consisted of six mice.

Male Wistar rats of initial body weight from 200 to $230 \mathrm{~g}$ were also used. The animals were housed in plastic cages in constant temperature facilities exposed to 12-12 light-dark cycle. Water and food were available ad libitum. The solution of nanoparticles was administered on the shaved skin of the abdomen.

The solution of nanoparticles was administered intraperitoneally (i.p.) - group no. 1 or intragastrically by means of an oral gavage (p.o.) - group no. 2. The volume of the test solution was $10 \mathrm{~mL} / \mathrm{kg}$. Nothing was given to the control groupgroup no. 3 .

All animal care and experimental procedures were carried out in accordance with European Union and Polish legislation acts concerning animal experimentation and approved by the Local Ethics Committee at the Jagiellonian University in Krakow.

\section{Acute toxicity, plasma, and tissue collection}

After administration of tested solution, mice were placed into the home cages and observed. The percent of death and body weight changes were determined during $24 \mathrm{~h}$. Subsequently, heparin $(500 \mathrm{j} / \mathrm{mice})$ and thiopental $(70 \mathrm{mg} / \mathrm{kg}$ i.p.) for each 
animal was administrated. After $20 \mathrm{~min}$, blood was collected from left common carotid artery. The animal spinal cord was interrupted, cut, and organs, namely the liver, kidneys, heart, brain, and lungs were weighted to determine the amount of nanosilver.

\section{Topical toxicity and skin collection}

Thiopental (70 mg/kg i.p.) was administrated to each animal. After $20 \mathrm{~min}$, the abdominal skin was smeared with depilatory cream (Joanna Sensitive, Poland), and 10 min later, the skin was wiped using a cotton swab moistened with $0.9 \%$ saline. The solution of nanoparticles $(0.3 \mathrm{ml} /$ animals $)$ was administered on the skin. The percent of death and body weight changes were determined during 24 h. Subsequently, thiopental (70 mg/kg i.p.) was administrated to each animal. After $20 \mathrm{~min}$, the skin was collected.

\section{Statistical analysis}

The results obtained were analyzed using a two-way analysis of variance (ANOVA), followed by a Bonferroni post-hoc test with the significance level set at 0.05 (body weight changes). The outcomes were expressed as the means \pm standard error of the mean (SEM). GraphPad Prism 6.0 was used for data analysis.

\section{Metal concentration determination}

The biodistribution of AgNPs was investigated in selected organs. For this purpose, after 24 h, the liver, kidneys, heart,
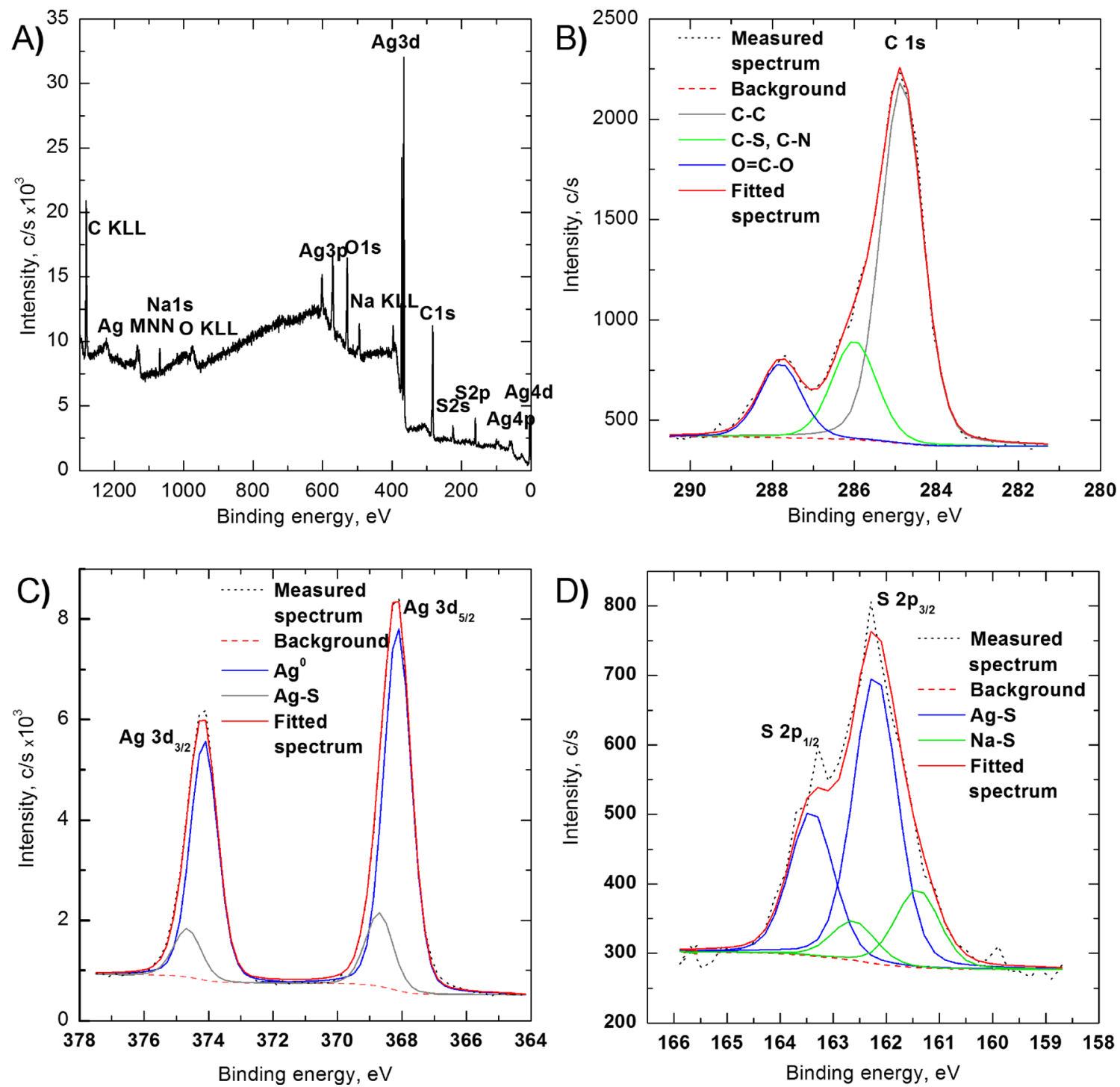

Fig. 1 Survey scan performed with X-ray photoelectron spectroscopy of AgNPs (a). XPS spectra with fitted lines C 1s (b), Ag 3d (c), and S 2p (d) 

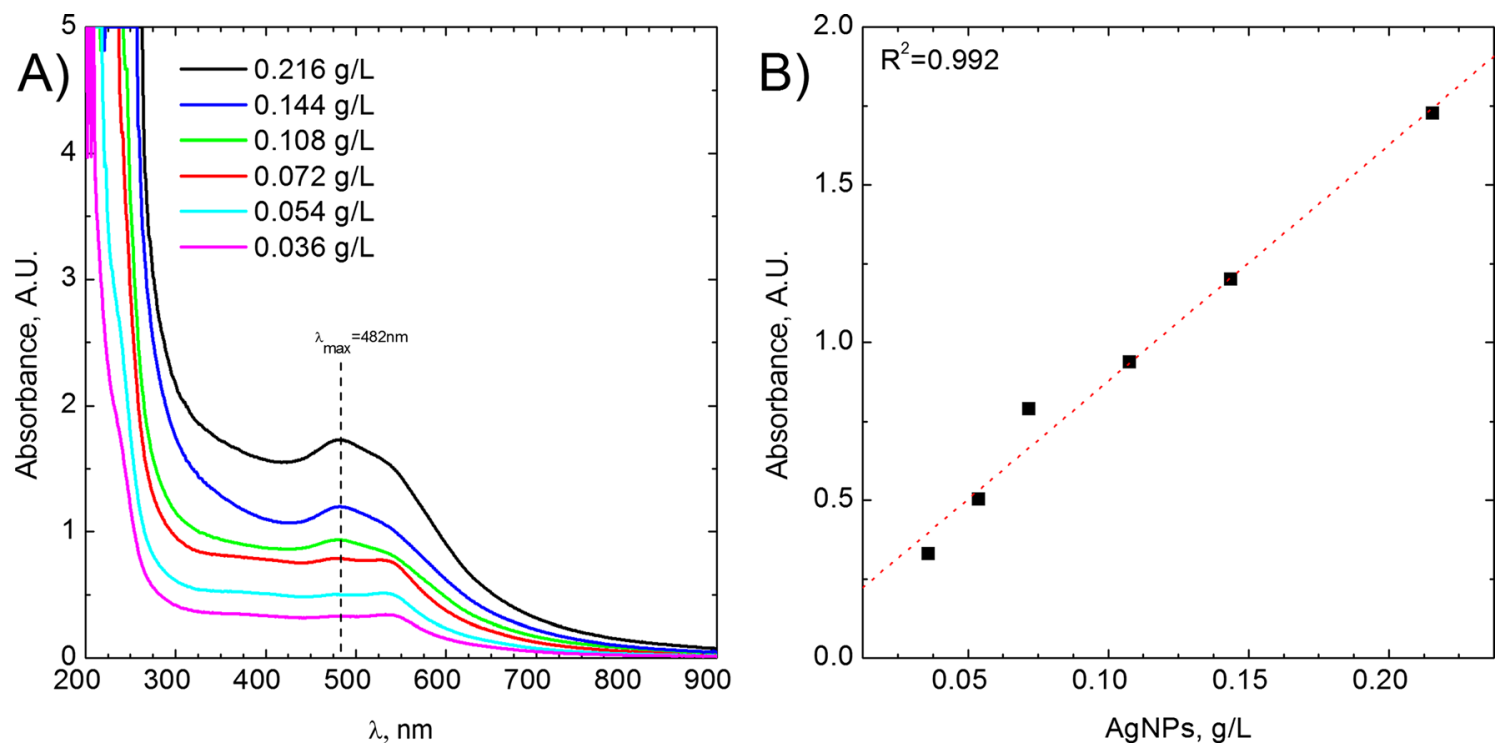

Fig. 2 a UV-Vis spectrum of obtained AgNPs. b Determination of absorption coefficient

brain, and lungs were collected to determine the amount of nanosilver. Then, these organs were weighted and mineralized to extract silver. The extraction was performed according to protocol described in our previous papers (Wojnicki et al. 2013; Bednarski et al. 2015). The metal concentration was performed using spectrometer a 4200MP-AES Agilent.

\section{Results}

\section{AgNP characterization}

X-ray photoelectron spectroscopy (XPS) of AgNPs confirmed the presence of silver nanoparticles with cysteine conjugated to the surfaces of the nanoparticles. The survey scan also indicated the presence of $\mathrm{Ag}, \mathrm{O}, \mathrm{C}, \mathrm{S}, \mathrm{N}$, and $\mathrm{Na}$ elements in the sample (Fig. 1a). The atomic concentrations of each element were determined to be as the following: $\mathrm{C} 1 \mathrm{~s}-54.56 \% ; \mathrm{N} 1 \mathrm{~s}$ $6.05 \%$; O 1s-21.29\%; S 2p-5.24\%; Ag 3d-10.71\%; and $\mathrm{Na} 1 \mathrm{~s}-2.15 \%$. Detailed analysis of selected elements and bound type in AgNPs is shown in Fig. 1b-d. Figure 1b shows spectrum of $\mathrm{C} 1 \mathrm{~s}$ with fitted peaks that come from cysteine bounded to nanoparticle surfaces: peak at $286.0 \mathrm{eV}$ corresponds to $\mathrm{C}-\mathrm{N}$ and $\mathrm{C}-\mathrm{S}$ bonds while at $287.8 \mathrm{eV}$ comes from $\mathrm{O}-\mathrm{C}=\mathrm{O}$ group in cysteine structure (Cavalleri et al. 2001). Ag 3d spectrum (Fig. 1c) can be fitted by two doublets with binding energies of the $\mathrm{Ag} 3 \mathrm{~d} 5 / 2$ component at 368.2 and $368.7 \mathrm{eV}$ (DS = $6.0 \mathrm{eV}$ ), respectively. First peak is attributed to metallic $\mathrm{Ag}$, while the second is attributed to Ag-S from nanoparticlecysteine bonds (Tong et al. 2015; Ferraria et al. 2012). These
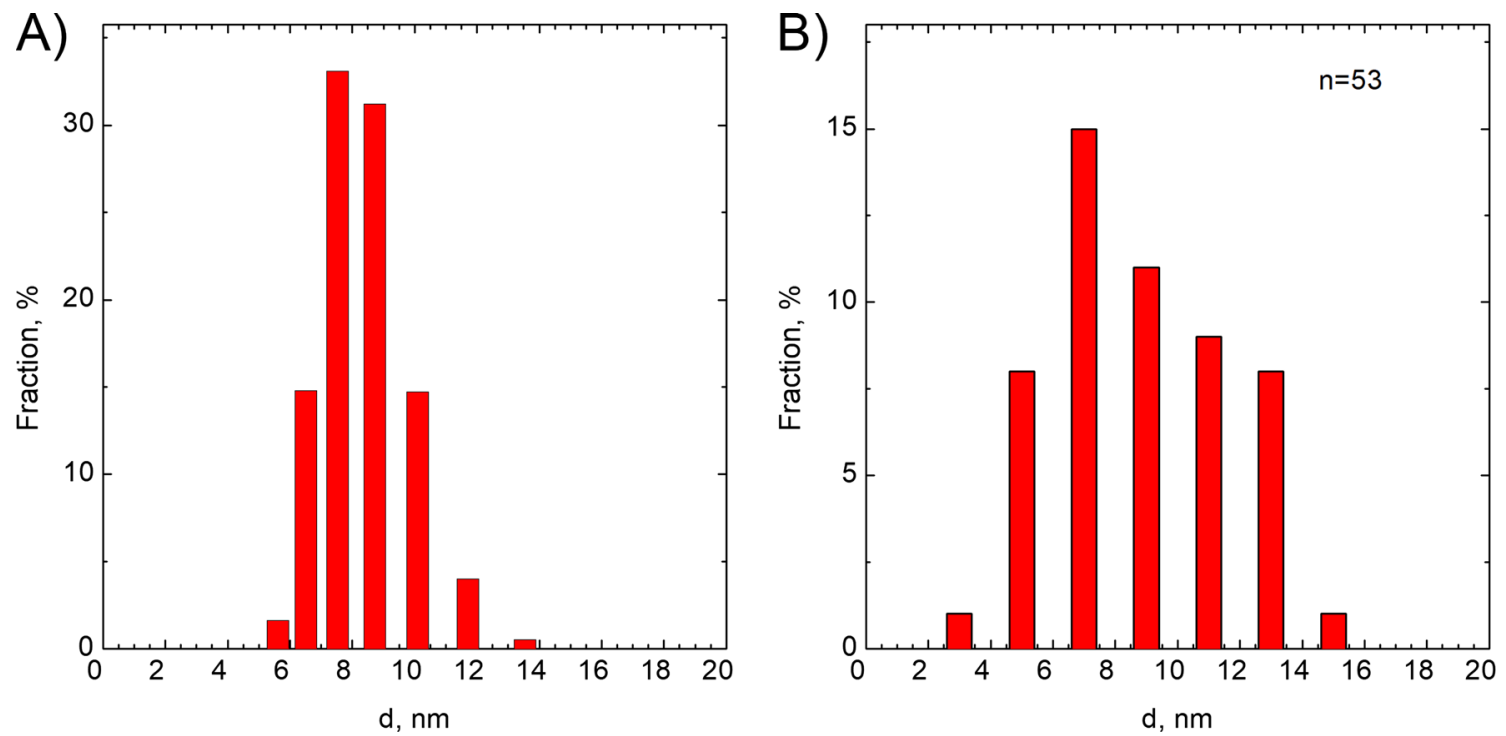

Fig. 3 Particle size distribution determined using a DLS method and b HR-TEM image analysis method 
results confirmed that cysteine was chemically bonded to AgNP surfaces. Figure $1 d$ shows S $2 p$ spectrum. Peak deconvolution revealed presence of two chemical states of sulfur at 161.4 and $162.6 \mathrm{eV}$ for S 2p3/2. The first corresponds to $\mathrm{Na}-\mathrm{S}$ bond formed by cysteine in alkaline solution. This suggests that cysteine could be also physically adsorbed on the particle surfaces. The second peak comes from Ag-S bonds between particle and cysteine (Battocchio et al. 2014).

It is well known that the silver sulfide is a semiconductor, and its presence in the system should be observable in the UVVis spectra. In the case of AgNPs, their presence can also be confirmed thanks to plasmon resonance peak. Therefore, UVVis analyses were performed, and results obtained are shown in Fig. 2a.

In Fig. 2a, UV-Vis spectra of the solutions are shown. It has to be underlined that these spectra were recorded for diluted samples. The samples were diluted from 50 to 300 times, respectively. As it can be seen, one asymmetrical peak at the wavelength of $482 \mathrm{~nm}$ can be observed. This peak can be attributed to surface plasmon resonance (Darroudi et al. 2010). The red shift of plasmon resonance might be related to L-cysteine molecule on the surface of AgNPs (Knauer et al. 2013). It should be noted that UV-Vis analysis did not confirm the presence of $\mathrm{Ag}_{2} \mathrm{~S}$.

The absorption coefficient of synthetized colloid can be calculated taking into account the assumption that the absorbance is a linear function of the colloid concentration, according to Lambert-Beer's law. From the slope of colloid concentration vs. absorbance, the absorption coefficient can be determined (see Fig. 2b). The slope is equal to $7.49 \pm 0.48 \frac{L}{\mathrm{~g} \times \mathrm{cm}}$, and the intercept is $0.13 \pm 0.06$.

Particle size and size distribution as well as zeta potential were determined using a Malvern instrument. The zeta

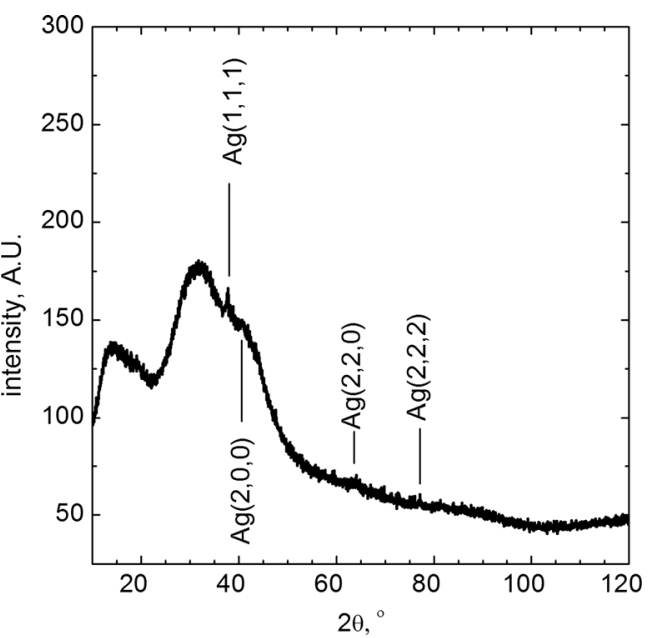

Fig. 5 XRD pattern of obtained AgNPs

potential determined using this equipment is equal to -50.6 $\pm 4.47 \mathrm{mV}$. Strong negative zeta potential is probably related to the zwitterionic properties of L-Cysteine (Quesada-Moreno et al. 2014). Deprotonation of L-cysteine depends on $\mathrm{pH}$. Two forms are present in the system at a $\mathrm{pH}$ of 7 . The first one is the zwitterion and anionic form. Moreover, using DLS method, particle size and size distribution were determined. The results are shown in Fig. 3a. To make it clear, in Fig. 3b, particle size distribution determined using HR-TEM images is also shown.

As it can be seen, the main fraction is of about $8 \mathrm{~nm}$ in diameter using DLS as well as HR-TEM image analysis method. It should also be noted that the distribution of the particle size is symmetrical, whereas typical methods of nanoparticle synthesis result in log-normal particle size distribution. In case of HR-TEM image analysis method, particle size distribution seems to be wider and is equal to ca. $3.2 \mathrm{~nm}$. During HR-TEM image analysis, 53 particles were analyzed $(n=53)$.
Fig. 4 HR-TEM analysis (a) and selection of polycrystal structure (b) FFT analysis of single crystal (c)

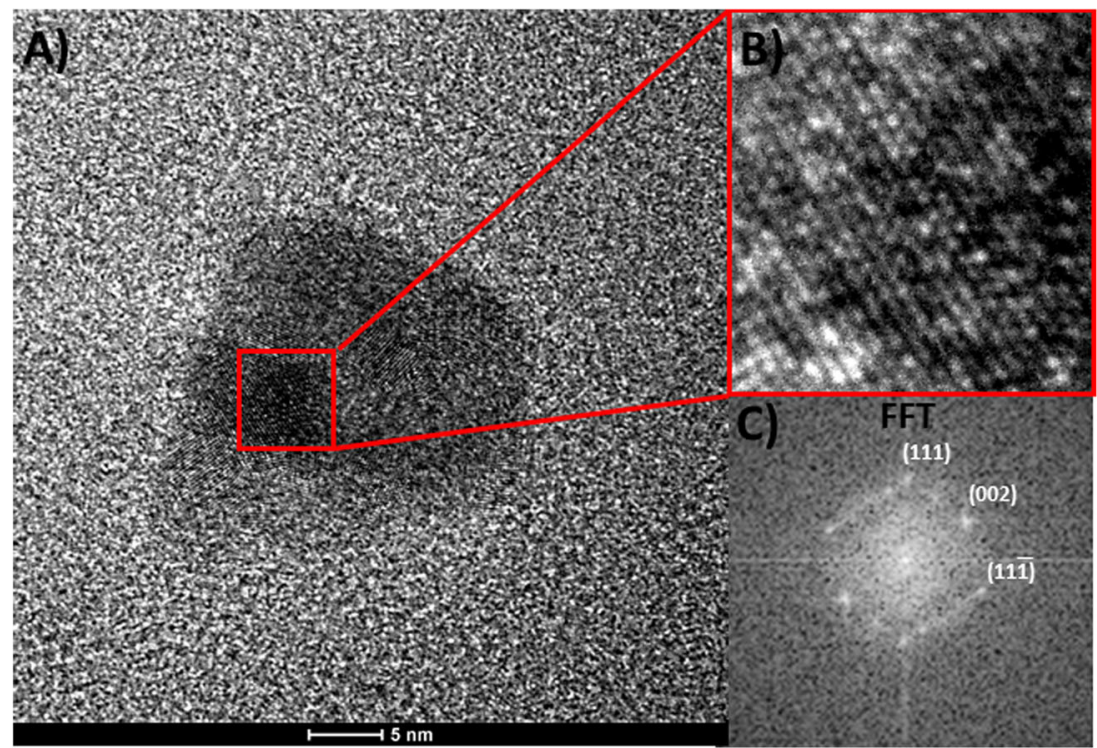




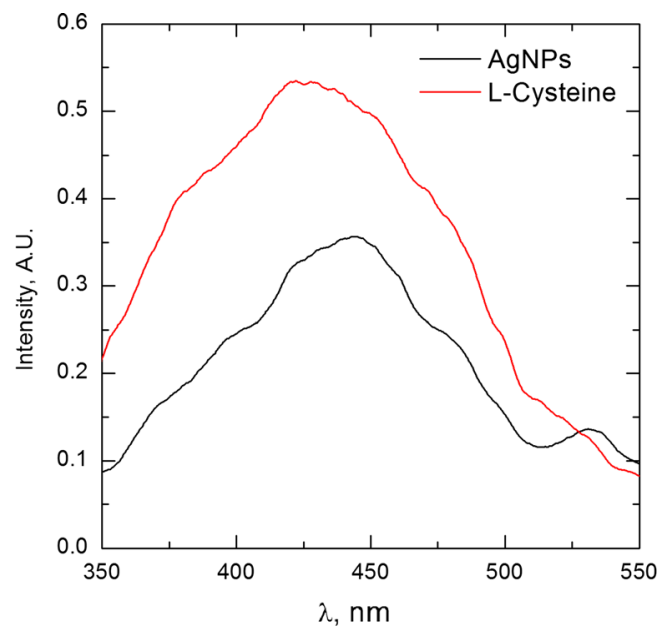

Fig. 6 Fluorescence of AgNPs functionalized with L-cysteine

The HR-TEM analysis was performed (Fig. 4a); the particle size was comparable to that determined using DLS method.

Moreover, FFT analysis of a selected picture (red square in Fig. 4a) was performed to obtain the information about crystallography of synthetized material (Fig. 4c). Selected crystal plane are marked on the diffractogram. The angle between planes (111) and (002) is equal to $54^{\circ}$, which is in agreement to the literature. However, it should be underlined that obtained nanoparticles have polycrystalline structure.

The results of further analyses of AgNPs carried out using XRD are shown in Fig. 5.

There is only one sharp peak. Low intensity of this peak is related to the particle size. As it was shown in Fig. 4a, obtained nanoparticles have polycrystal structure, where single grain size is significantly lower than $3 \mathrm{~nm}$. Therefore, the peak broadening is observed which is also related to the
Scherrer's equation. Unfortunately, neither HR-TEM analysis nor XRD analysis confirmed the presence of $\mathrm{Ag}_{2} \mathrm{~S}$ in the synthetized material. This might be related to the thickness of $\mathrm{Ag}_{2} \mathrm{~S}$ layer. In the presence of silver sulfide on the surface of AgNPs, an additional optical effect should be observed, related directly to the semiconducting properties of $\mathrm{Ag}_{2} \mathrm{~S}$. That was the reason why the samples were also analyzed using fluorescence method. It is well known that silver sulfide exhibits quantum dots properties. Xiulan Wu et.al (2015) have shown that for spherical $\mathrm{Ag}_{2} \mathrm{~S}$ nanoparticles of $5 \mathrm{~nm}$ in diameter, the maximum emission peak appears at the wavelength of ca. $750 \mathrm{~nm}$, after excitation at $520 \mathrm{~nm}$. It should also be underlined that $\mathrm{Ag}_{2} \mathrm{~S}$ nanoparticles obtained by Xiulan $\mathrm{Wu}$ et al. were also functionalized using L-cysteine (Fig. 6).

As it can be seen from Fig. 6, the fluorescence of the sample is related to L-cysteine present on the surface of AgNPs. Insignificant changes in the left shoulder of the fluorescence curve of AgNPs are related to the absorption bend of metallic silver, as it is shown in Fig. 2a. There is no fluorescence above $600 \mathrm{~nm}$. However, a small peak at $525 \mathrm{~nm}$ appears. This suggests that $\mathrm{Ag}-\mathrm{S}$ bonding visible in XPS (see Fig. 1c) is related to the chemisorption of L-cysteine on the surface of AgNPs.

\section{Body weight changes in experimental animals}

There were no significant changes in body weight of mice $24 \mathrm{~h}$ after intraperitoneal (i.p.) administration of the test solution in comparison to changes of body weight in mice from the control group ( $p=0.0621)$. After intragastric (p.o.) administration, the changes of the body weight of tested mice were significantly lower in comparison to the body weight changes stated in mice $(p=0.0398)$. These results are shown in Fig. 7.
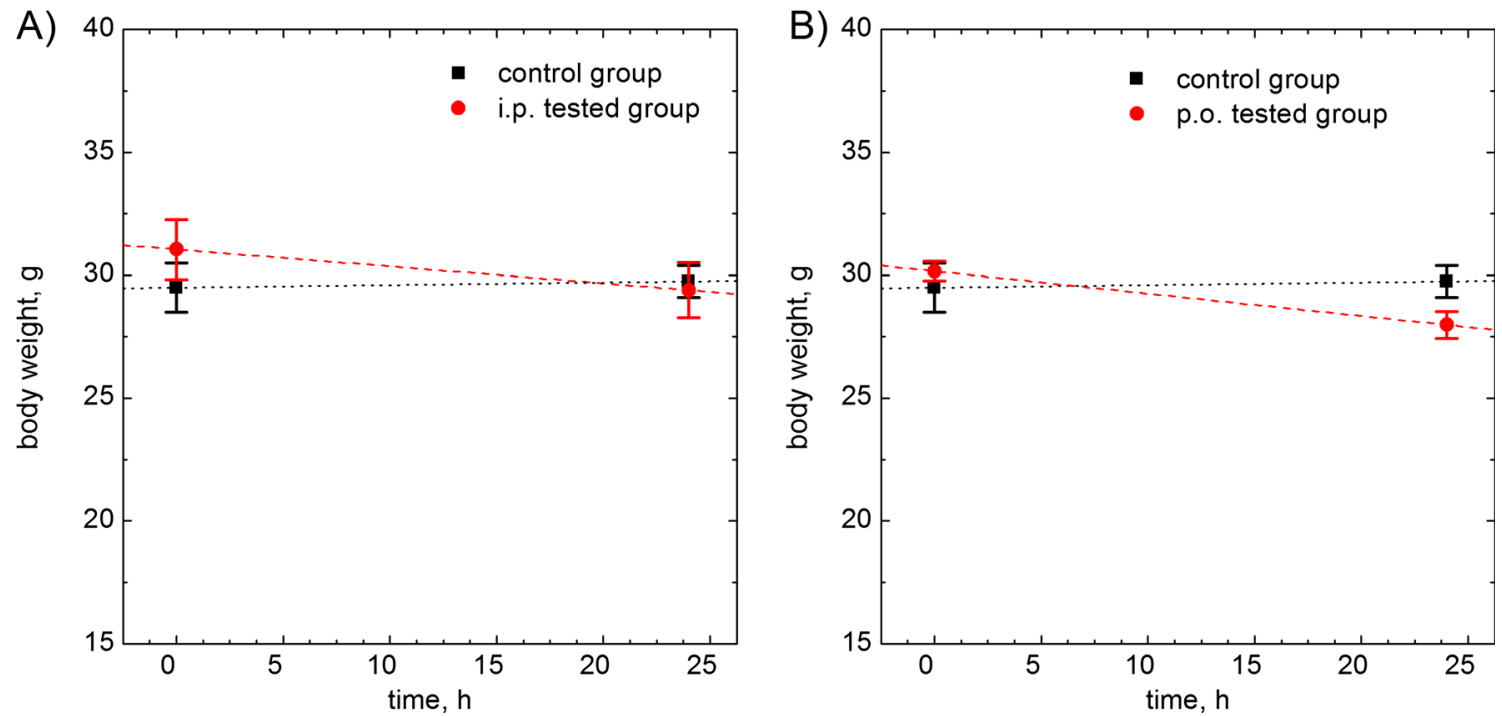

Fig. 7 Body weight changes. a Comparison of control group to tested group after intraperitoneal administration. b Comparison of control group to tested group after intragastric administration. Mean $\pm \mathrm{SEM}, n=6$. Statistical significance: $* p<0.05$ (two-way ANOVA) 
Table 1 AgNP biodistribution in selected organs in percentage of given dose

\begin{tabular}{llll}
\hline Brain & Kidneys & Liver & Blood \\
\hline After P.O administration & & & \\
$1.82 \times 10^{-2} \pm 5.79 \times 10^{-3}$ & $1.55 \times 10^{-2} \pm 2.21 \times 10^{-3}$ & $6.53 \times 10^{-2} \pm 2.87 \times 10^{-2}$ & $4.16 \times 10^{-2} \pm 1.51 \times 10^{-2}$ \\
After I.P administration & & & $9.45 \times 10^{-2} \pm 5.46 \times 10^{-2}$ \\
$3.59 \times 10^{-2} \pm 1.52 \times 10^{-3}$ & $2.98 \times 10^{-1} \pm 6.24 \times 10^{-2}$ & $2.93 \pm 4.31 \times 10^{-1}$ & 9.45 \\
\hline
\end{tabular}

\section{AgNP acute toxicity and biodistribution}

Acute toxicity is extremely important parameter for new drug safety. Thus, two ways of AgNP administration were chosen. The first one was intraperitoneal, and the second one was intragastric. During these trials, sedation of animals was noted. However, there was no death during the first $24 \mathrm{~h}$. It can be concluded that synthesized AgNPs do not exhibit acute toxicity after intragastric and intraperitoneal administration.

Then, the mice were sacrificed and selected organs were removed for further analyses. The results of silver nanoparticles biodistributions are gathered and shown in Table 1.

The amount of AgNPs determined in selected organs is low. Only in the case of intraperitoneal administration, a significant accumulation in the liver was stated. The absorption from the digestive tract is very low. These results are comparable to our previous report (Bednarski et al. 2015). However, it should be stressed that the amount of AgNPs absorbed from the digestive tract is about two times higher in comparison to gold nanoparticles (Bednarski et al. 2015) .

\section{Irritating test}

Additionally, an irritating test on the rate skin was performed. About $300 \mu \mathrm{L}$ of AgNPs suspension was rubbed into the skin of rats $(n=3)$. After $24 \mathrm{~h}$, no irritating effect was observed. Moreover, the presence of the silver in the blood was not detected. This in turn suggests that dermal absorption also does not occur, which indicates that AgNPs can be safely used in cosmetics.

\section{Conclusions}

The antibacterial properties of silver nanoparticles are well known. In this paper, a simple and effective method for silver nanoparticle synthesis was described. These nanoparticles showed excellent biocompatibility. Thanks to the application of L-cysteine silver complex, it was possible to obtain the concentration of colloid equal to up to $10.7 \mathrm{~g} / \mathrm{L}$. Such a high concentration of colloid is extremely difficult to synthesize in one step. The only requirement for the developed method is the purification process, in order to remove leftover of reductant and L-cysteine.
Moreover, narrow particle size distribution of the colloid makes its behavior in living organism easy to investigate. Since the properties of nanoparticles depend on their size, in the case of polydisperse samples, the assessment of the biological effect can be fuzzy as different fractions can give different responses. Finally, it is worth mentioning that L-cysteine was applied as a stabilizing agent. This simple amino acid can be easily used to link an antibody or any other compound to the surface of nanoparticles.

Funding information This study was funded by the Polish Ministry of Science and Education (grants nr IP2014051773 Iuventus Plus).

Compliance with ethical standards All animal care and experimental procedures were carried out in accordance with European Union and Polish legislation acts concerning animal experimentation and approved by the Local Ethics Committee at the Jagiellonian University in Krakow.

Conflict of interest The authors declare that they have no conflict of interest.

Open Access This article is distributed under the terms of the Creative Commons Attribution 4.0 International License (http:// creativecommons.org/licenses/by/4.0/), which permits unrestricted use, distribution, and reproduction in any medium, provided you give appropriate credit to the original author(s) and the source, provide a link to the Creative Commons license, and indicate if changes were made.

\section{References}

Battocchio C, Fratoddi I, Fontana L, Bodo E, Porcaro F, Meneghini C, Pis I, Nappini S, Mobilio S, Russo MV, Polzonetti G (2014) Silver nanoparticles linked by a Pt-containing organometallic dithiol bridge: study of local structure and interface by XAFS and SRXPS. Phys Chem Chem Phys 16(23):11719-11728. https://doi. org/10.1039/C4CP01264J

Bednarski M, Dudek M, Knutelska J, Nowiński L, Sapa J, Zygmunt M, Nowak G, Luty-Błocho M, Wojnicki M, Fitzner K, Tęsiorowski M (2015) The influence of the route of administration of gold nanoparticles on their tissue distribution and basic biochemical parameters: in vivo studies. Pharmacol Rep 67(3):405-409. https://doi.org/10. 1016/j.pharep.2014.10.019

Cavalleri O, Oliveri L, Daccà A, Parodi R, Rolandi R (2001) XPS measurements on 1-cysteine and 1-octadecanethiol self-assembled films: a comparative study. Appl Surf Sci 175-176:357-362

Cobley CM, Chen J, Cho EC, Wang LV, Xia Y (2011) Gold nanostructures: a class of multifunctional materials for biomedical 
applications. Chem Soc Rev 40(1):44-56. https://doi.org/10.1039/ B821763G

Darroudi M, Ahmad MB, Abdullah AH, Ibrahim NA, Shameli K (2010) Effect of accelerator in green synthesis of silver nanoparticles. Int J Mol Sci 11(10):3898-3905. https://doi.org/10.3390/ijms11103898

Ferraria AM, Carapeto AP, Botelho do Rego AM (2012) X-ray photoelectron spectroscopy: silver salts revisited. Vacuum 86(12):19881991. https://doi.org/10.1016/j.vacuum.2012.05.031

Galdiero S, Falanga A, Vitiello M, Cantisani M, Marra V, Galdiero M (2011) Silver nanoparticles as potential antiviral agents. Molecules 16(10):8894-8918. https://doi.org/10.3390/molecules16108894

Guo Z, Sadler PJ (1999) Metals in medicine. Angew Chem Int Ed 38(11): 1512-1531. https://doi.org/10.1002/(SICI)1521-3773(19990601) 38:11<1512::AID-ANIE1512>3.0.CO;2-Y

Huang X, El-Sayed IH, Qian W, El-Sayed MA (2006) Cancer cell imaging and photothermal therapy in the near-infrared region by using gold nanorods. J Am Chem Soc 128(6):2115-2120. https://doi.org/ $10.1021 / \mathrm{ja} 057254 \mathrm{a}$

Knauer A, Schneider S, Möller F, Csáki A, Fritzsche W, Köhler JM (2013) Screening of plasmonic properties of composed metal nanoparticles by combinatorial synthesis in micro-fluid segment sequences. Chem Eng J 227(0):80-89. https://doi.org/10.1016/j.cej. 2012.10.008

Kokura S, Handa O, Takagi T, Ishikawa T, Naito Y, Yoshikawa T (2010) Silver nanoparticles as a safe preservative for use in cosmetics. Nanomed Nanotechnol Biol Med 6:570-574
Luty-Błocho M, Wojnicki M (2015) Single-step synthesis of onion-like $\mathrm{Au}-\mathrm{Pd}-\mathrm{PtNPs}$ nanoparticles using microflow system. J Flow Chem 5(4):197-200. https://doi.org/10.1556/1846.2015.00026

Luty-Błocho M, Wojnicki M, Grzonka J, Kurzydłowski KJ (2014) The synthesis of stable platinum nanoparticles in the microreactor. Arch Metall Mater 59(2):509-512

Quesada-Moreno MM, Avilés-Moreno JR, Márquez-García AA, LópezGonzález JJ (2014) Deducing the molecular properties of zwitterionic, protonated, deprotonated, and double-deprotonated forms of L-cysteine from vibrational spectroscopy (IR, Raman, VCD) and quantum chemical calculations. J Mol Model 20(6):1-15

Tong J, Wang YJ, Wang Z, Sun JZ, Tang BZ (2015) Crystallizationinduced emission enhancement of a simple tolane-based mesogenic luminogen. J Phys Chem C 119(38):21875-21881. https://doi.org/ 10.1021/acs.jpcc.5b06088

Wojnicki M, Luty-Blocho M, Bednarski M, Dudek M, Knutelska J, Sapa J, Zygmunt M, Nowak G, Fitzner K (2013) Tissue distribution of gold nanoparticles after single intravenous administration in mice. Pharmacol Rep 65(4):1033-1038. https://doi.org/10.1016/S17341140(13)71086-7

Wojnicki M, Zabieglińska K, Luty-Błocho M (2015) Influence of experimental condition on silver nanoparticle synthesis process in aqueous solutions. Ores Non-Ferrous Metals 60(3):103-109

Wu X, Liao L, Du W, Qin A (2015) Near infrared emission of Ag2S quantum dots and their fluorescence quenched by gold nanoparticles. Proc Eng 102:273-277. https://doi.org/10.1016/j.proeng.2015. 01.143 